\title{
Coadsorption phases of CO and oxygen on Pd(111) studied by scanning tunneling microscopy
}

\author{
Javier Méndez, ${ }^{1,2, *}$ Sang Hoon Kim,,${ }^{1, \dagger}$ Jorge Cerdá, ${ }^{2}$ Joost Wintterlin, ${ }^{1, \$}$ and Gerhard Ertl ${ }^{1}$ \\ ${ }^{1}$ Fritz-Haber-Institut der Max-Planck-Gesellschaft, D-14195 Berlin, Germany \\ ${ }^{2}$ Instituto de Ciencia de Materiales de Madrid CSIC, E-28049 Madrid, Spain
}

(Received 3 August 2004; published 9 February 2005)

\begin{abstract}
The adsorption of $\mathrm{CO}$ on an oxygen precovered $\mathrm{Pd}(111)$ surface was investigated between 60 and $300 \mathrm{~K}$. Applied methods were variable temperature scanning tunneling microscopy (STM) and video STM to analyze the coadsorption structures. The STM data are compared with simulated STM images for the various surface phases in order to identify the appropiate structural model for each case. Low-energy electron diffraction and reaction isotherms by means of mass spectrometry were used to correlate the phases with the reaction yielding $\mathrm{CO}_{2}$. The video-STM data recorded during $\mathrm{CO}$ adsorption at $300 \mathrm{~K}$ on the $(2 \times 2)_{\mathrm{O}}$ phase show a fast phase transition into the $(\sqrt{3} \times \sqrt{3}) R 30_{\mathrm{O}}^{\circ}$ structure, followed by reaction to $\mathrm{CO}_{2}$. The reaction only starts after completion of the phase transition, indicating that the $(\sqrt{3} \times \sqrt{3}) R 30_{\mathrm{O}}^{\circ}$ structure plays a crucial role for the reaction. At temperatures between 170 and $190 \mathrm{~K}$ the phase transition is slow enough to be monitored with STM. The experimental images of both the $(2 \times 2)_{\mathrm{O}}$ and the $(\sqrt{3} \times \sqrt{3}) R 30_{\mathrm{O}}^{\circ}$ structures are well reproduced by the simulations. Further $\mathrm{CO}$ adsorption caused a second phase transition into a $p(2 \times 1)_{\mathrm{O}}$ structure. The STM simulations strongly support a pure oxygen $p(2 \times 1)$ structure, rather than a mixed $\mathrm{O}+\mathrm{CO}$ structure, in contrast to previous experimental work. The $\mathrm{CO}$ molecules form the same structures between the $\mathrm{O}$ islands that are known from the pure $\mathrm{Pd}(111) / \mathrm{CO}$ system. At lower temperatures, between 110 and $60 \mathrm{~K}$, a so far unknown $(2 \times 2)$ phase was observed. The formation of this structure, and its imaging by the STM, show that it constitutes a mixed $p(2 \times 2)_{\mathrm{O}+\mathrm{CO}}$ structure, where the oxygen atoms remain unchanged, and the $\mathrm{CO}$ molecules occupy hcp sites between the $\mathrm{O}$ atoms.
\end{abstract}

DOI: 10.1103/PhysRevB.71.085409

PACS number(s): 68.43.Fg, 68.43.Hn, 68.37.Ef, 71.15.Mb

\section{INTRODUCTION}

One important aspect in surface catalytic reactions is the structures and phases formed by the adsorbed reactants under reaction conditions. Adsorption phases determine the binding energies of the reactants, their relative orientations, and may lead to transport processes. The mostly applied Langmuirian "checkerboard" model, which assumes identical adsorption sites of the reactant particles on the surface and a statistical lattice-gas occupation of these sites, neglects this aspect. In particular, investigations by scanning tunneling microscopy (STM) have shown that this model is a serious oversimplification. ${ }^{1}$ For example, one of the most simple catalytic reactions, the oxidation of $\mathrm{CO}$ on $\mathrm{Pt}(111)$, is characterized at low temperatures by a two-dimensional (2D) phase separation of $\mathrm{CO}$ molecules and $\mathrm{O}$ atoms, which limits the reaction to the phase boundaries.,3 Under realistic conditions, such effects are most likely still important when the steady-state coverages are high.

A similar system, $\mathrm{Pd}(111)$ with adsorbed $\mathrm{CO}$ and $\mathrm{O}$, is much richer in surface phases. The oxygen layer in this system can be compressed, and there is a large diversity of $\mathrm{CO}$ structures, ${ }^{4,5}$ so that one can expect an even more complicated reaction scenario than on $\mathrm{Pt}(111)$. For oxygen on $\operatorname{Pd}(111)$, two different phases have been observed, a $p(2$ $\times 2)_{\mathrm{O}}$ and a $(\sqrt{3} \times \sqrt{3}) R 30_{\mathrm{O}}^{\circ}$ structure. ${ }^{6-10}$ The former is obtained by room-temperature adsorption of $\mathrm{O}_{2}$, the latter by oxygen exposure to an oxygen precovered surface at $270 \mathrm{~K}$ or by post dosing other adsorbates, such as $\mathrm{CO} .{ }^{11}$ For $\mathrm{CO}$ on $\operatorname{Pd}(111)$, at least 17 different structures have been reported $^{4-6,12-20}$ ranging from a $(\sqrt{3} \times \sqrt{3}) R 30_{\mathrm{CO}}^{\circ}$ structure with a coverage of $\theta=1 / 3$, to a $p(2 \times 2)_{\mathrm{CO}}$ structure with $\theta$ $=3 / 4$. Two models have been discussed that describe the transitions between the various CO structures: (i) a continuous one, according to which the CO layer is more or less continuously compressed and (ii) a discrete one that assumes separate domains with $\mathrm{CO}$ molecules at different distances, but on defined adsorption sites. Recent work favored the latter, in which the transitions occur by changes of the domain widths. ${ }^{20}$

$\mathrm{CO}$ adsorption on the $\mathrm{O}$ covered surface leads to a compression of the $p(2 \times 2)_{\mathrm{O}}$ phase into the $(\sqrt{3} \times \sqrt{3}) R 30_{\mathrm{O}}^{\circ}$ structure, ${ }^{7,11,21}$ and then into a $p(2 \times 1)$ phase. ${ }^{22}$ The latter was proposed to be a mixed $p(2 \times 1)_{\mathrm{O}+\mathrm{CO}}$ structure with alternating rows of $\mathrm{CO}$ molecules and oxygen atoms, ${ }^{7}$ but density functional theory (DFT) calculations indicated that separate $p(2 \times 1)_{\mathrm{O}}$ and $p(2 \times 1)_{\mathrm{CO}}$ domains are energetically more favorable. ${ }^{21}$

In this paper we report an investigation of the different phases that occur during coadsorption of $\mathrm{CO}$ and oxygen on the $\mathrm{Pd}(111)$ surface by means of STM. The findings are correlated with mass spectrometer (MS) data and low-energy electron diffraction (LEED) measurements in order to explore the roles of the structures in the catalytic reaction. In addition, we report a metastable mixed $p(2 \times 2)_{\mathrm{CO}+\mathrm{O}}$ structure obtained at low temperatures. The atomic structures of all observed phases are further investigated by combined $a b$ initio total energy calculations and STM simulations.

\section{EXPERIMENTAL}

The experimental apparatus consisted of an ultrahigh vacuum (UHV) chamber, equipped with LEED optics, an 
Auger electron spectrometer, a quadrupole mass spectrometer, and further UHV facilities. In addition to a turbomolecular, ion and sublimation pumps, a nonevaporable getter (NEG) pump was employed that has a high sorption rate for hydrogen and reduced the hydrogen pressure. Gases were introduced into the chamber through leak valves, and high purity gases $(99.999 \%)$ were used.

The microscope was a variable temperature beetle-type STM cooled by liquid He. In some experiments a pocket-size STM (Ref. 23) with fast scanning "video" mode ${ }^{24}$ was also used. STM tips were made from tungsten or iridium. Both types of tips were self-sputtered ${ }^{25}$ and treated by field emission before STM experiments. In this way contaminants and tip effects during reactions were reduced.

Mass spectra were recorded with a quadrupole mass spectrometer (QMS) in line-of-sight geometry. A metallic shield around the QMS reduced the spurious $\mathrm{CO}_{2}$ signal coming from the chamber and gases entered through an aperture in front of the sample. The aperture diameter was smaller than the sample diameter, but the distance to the sample was chosen not too small $(\sim 5 \mathrm{~mm})$ to avoid shadowing of the surface during $\mathrm{CO}$ adsorption. The data were recorded by measuring the $\mathrm{CO}_{2}$ signal when the O-covered surface was exposed to different $\mathrm{CO}$ pressures at constant temperatures. The integrals of such plots were almost constant for different pressures of $\mathrm{CO}$, as expected for a fixed amount of $\mathrm{O}$. This fact is taken as evidence that the measured $\mathrm{CO}_{2}$ indeed mainly came from the reaction with the adsorbed oxygen on the sample. The dose of $\mathrm{CO}$ is calculated by multiplying the time axis by the corresponding $\mathrm{CO}$ pressure in Torr and considering a sticking coefficient of $1\left(1 \mathrm{~L}=1 \mathrm{~s}\right.$ at $10^{-6}$ Torr).

Before carrying out experiments, the chamber had to be cleaned with oxygen $\left(1 \times 10^{-6}\right.$ Torr oxygen for $\left.10 \mathrm{~min}\right)$. These treatments were necessary, because the first oxygen doses produced $\mathrm{CO}_{2}$ and hydrogen, probably mainly originating from the chamber walls. The hydrogen inhibited the adsorption of oxygen on the palladium surface while the $\mathrm{CO}_{2}$ strongly increased the background signal in the mass spectrometer data. This problem had also been noted by other authors. ${ }^{26}$ After several oxygen treatments, the $\mathrm{CO}_{2}$ signal from the chamber walls became smaller and could be subtracted from the $\mathrm{CO}_{2}$ signal as a background. The oxygen treatment, together with the NEG pump, also allowed to keep the hydrogen at low enough level to allow adsorption of oxygen on the sample.

The sample was a (111) oriented palladium single crystal. Preparation in UHV consisted of cycles of $\mathrm{Ar}^{+}$sputtering, heating in $2 \times 10^{-7}$ Torr oxygen at $720 \mathrm{~K}$ and flash annealing to $1110 \mathrm{~K}$, until a sharp $(1 \times 1)$ LEED pattern was observed. Fine cleaning was monitored with the mass spectrometer by means of temperature programmed desorption (TPD) after exposures of 10L of oxygen. For the clean surface, TPD spectra showed a main oxygen desorption at $850 \mathrm{~K}$, whereas $\mathrm{CO}$ desorption and the lack of the O-desorption peak indicated residual carbon contaminants.

The main experiments were performed starting with the oxygen-covered sample. For this purpose the sample was exposed to $2 \times 10^{-7}$ Torr oxygen during $50 \mathrm{~s}(10 \mathrm{~L})$ at temperatures between 230 and $300 \mathrm{~K}$. The resulting $p(2 \times 2)$ LEED indicated successful oxygen adsorption. Then the sample was exposed to $\mathrm{CO}$ at different pressures and temperatures. During gas exposures, the turbomolecular pump and/or the NEG pump were working to keep a low partial pressure of the residual gas.

For CO adsorption during the STM measurements, it turned out that the coverages in the STM images did not correspond to those measured by LEED or QMS, and the times necessary to complete the reaction were much longer when measured with STM. We ascribe this discrepancy to the tip shadowing effects, which lead to a lower local coverage under the tip. When the tip was retracted during dosing, the subsequent STM images showed the expected surface structures for the corresponding exposure times. The STM experiments shown here were obtained under both conditions, and they only differ in the appearance times of the structures. To reduce the shadowing effect when the tip was present, changing the scanning area or keeping a lateral drift was found to be helpful.

\section{THEORETICAL}

To resolve the atomic structures of the experimentally observed phases, total energy calculations were combined with STM simulations. For each proposed model, ab initio calculations have been performed relaxing the surface structure. Similar total energy calculations have also been performed for several tip structures (see below). However, the actual calculation of the STM images cannot be carried out at the $a b$ initio level since the coupled tip-sample STM system is computationally untractable (it would require a huge unit cell). Instead, we employ the extended Hückel theory ${ }^{27}$ (EHT) to construct the Hamiltonian of the entire STM setup. The EHT related parameters were obtained by fitting the EHT electronic structure to the DFT results, so that accurate parameters were independently obtained for each surface model and for each tip considered, as described below.

All total energy calculations were performed with the SIESTA-DFT-based code ${ }^{28}$ with the Troullier-Martins scheme $^{29}$ for the pseudopotential generation. For the STM simulations we used the Green's functions based code GREEN, ${ }^{30,31}$ which allows a fast evaluation of images and the possibility of explicitly considering different tips in order to address tip effects often seen in STM experiments. The code has been applied to a large variety of systems, typically providing a semiquantitative agreement with the experiment, which, in many cases, has allowed us to discriminate structural models. ${ }^{32,33}$

\section{A. DFT calculations for surfaces}

The surface structures were modeled by a twodimensional (2D) slab consisting of three Pd atomic planes, on top of which the adsorbates, $\mathrm{O}$ and/or $\mathrm{CO}$, where placed. For each phase the corresponding 2D lattice was employed. Only the atoms at the top Pd layer and the adsorbates were allowed to relax until forces were smaller than $0.05 \mathrm{eV} / \AA$, while the other two Pd layers were fixed at their bulk positions, at a lattice constant $a_{\mathrm{Pd}}=3.92 \AA$. Although the slab thickness is too small for an accurate evaluation of the total 
energies, we recall that the main purpose of these calculations is to obtain the equilibrium atomic coordinates together with a reasonable description of the electronic structure.

GGA (Ref. 34) pseudopotentials were generated using the atomic ground states as reference and taking as cutoff radii $r_{s}=r_{p}=2.25 \mathrm{Bohr}$ and $r_{d}=1.49 \mathrm{Bohr}$ for $\mathrm{Pd}, r_{s}=r_{p}=r_{d}$ $=1.01 \mathrm{Bohr}$ for $\mathrm{C}$ and $r_{s}=r_{p}=r_{d}=1.45 \mathrm{Bohr}$ for $\mathrm{O}$. Pseudocore corrections were also introduced for $\mathrm{Pd}$, with $r_{\text {core }}=1.0$ Bohr. The valence atomic orbital (AO) basis set employed in the SIESTA calculations comprised $2 s, 1 p$, and $2 d l$ shells for $\mathrm{Pd}$ and $2 s, 2 p$, and $1 d l$ shells for both $\mathrm{C}$ and $\mathrm{O}$; the spatial extents of the AOs were determined by using an energy cutoff of $200 \mathrm{meV}$. For the smallest unit cell investigated, the $(\sqrt{3} \times \sqrt{3}) R 30^{\circ}$ cell, $9 k$ points in the $2 \mathrm{D}$ Brillouin zone were used, for larger cells this number was reduced accordingly. A high accuracy was imposed to the three-center integrals evaluations by defining a fine grid in real space-we set the associated mesh cutoff to a value of $300 \mathrm{Ry}$.

\section{B. DFT calculations for STM tips}

We have performed calculations for both types of tips, since the experiments also employed two kinds of tips: W and Ir. For clean tips, we have found that the most relevant parameter determining the aspect of the images is related to the tip sharpness, rather than to its chemical identity. Therefore, we only present results for a sharp $\operatorname{Ir}(111)$ tip, the results for a sharp $\mathrm{W}(110)$ tip being qualitatively similar.

We modeled the tips by a 2D slab consisting of four Ir atomic planes oriented along the (111) direction of an fcc lattice. At the bottom of the slab a three-layer pyramid of ten Ir atoms was placed simulating the tip apex. The frontmost atom was either an Ir atom modeling a clean Ir tip [see inset in Fig. 1(b)] or an oxygen atom modeling an oxygen terminated tip [see inset in Fig. 1(c)]. A $p(3 \times 3)$ 2D lattice was used to minimize apex-apex interactions since, for the STM calculations described below, the apex is considered as isolated, i.e., nonperiodic.

The DFT calculations were this time carried out with the LDA approximation, ${ }^{35}$ in contrast to the GGA scheme employed for the surfaces. However, this is not a relevant issue since the actual tip-sample coupling is later calculated within the non-self-consistent EHT. The cutoff radii employed for the pseudopotential generation were $r_{s}=r_{p}=2.75 \mathrm{Bohr}, r_{d}$ $=2.00 \mathrm{Bohr}$, and $r_{\text {core }}=0.8 \mathrm{Bohr}$ for $\mathrm{Ir}$, and $r_{s}=r_{p}=r_{d}$ $=1.45 \mathrm{Bohr}$ for $\mathrm{O}$.

In the structure optimization, the ten apex atoms were allowed to relax, while all other atoms were kept fixed at the Ir-fcc bulk positions with $a_{\mathrm{Ir}}=3.87 \AA$. The valence AO basis set employed in SIESTA comprised $2 s, 1 p$, and $1 d l$ shells for Ir and $2 s, 2 p$, and $1 d l$ shells for $\mathrm{O}$; the spatial extents of the AOs were determined by using an energy cutoff of $100 \mathrm{meV}$. Nine $k$ points in the 2D Brillouin zone were used. Other parameters were set to the same values employed in the surfaces' calculations.

\section{EHT parametrizations}

As indicated above, for the calculation of the STM current and the generation of the STM images, we replaced the self- (a)

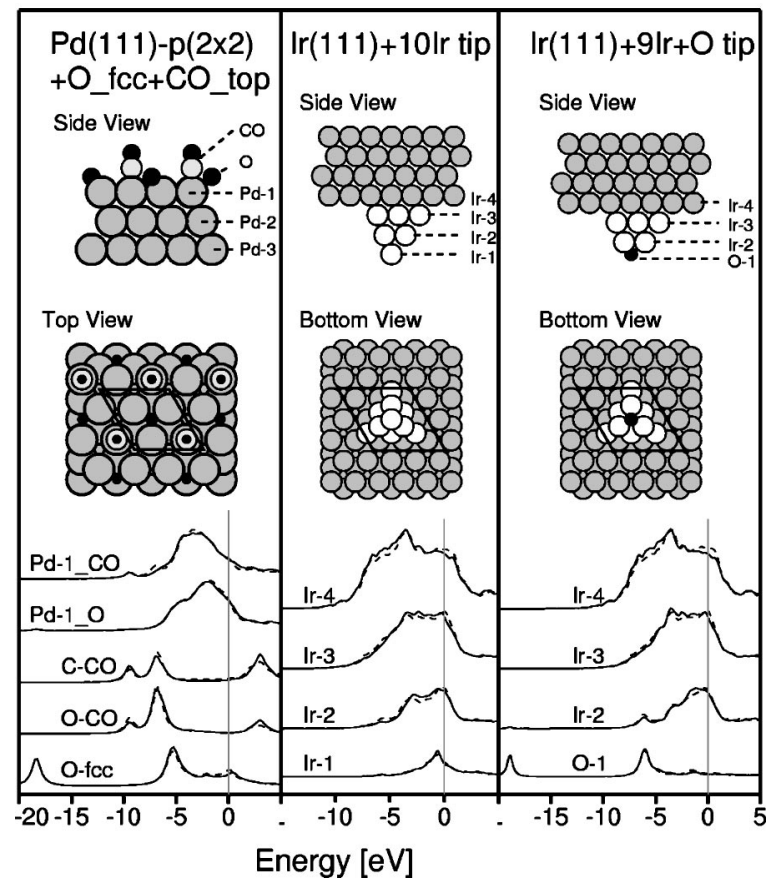

FIG. 1. Density of states (DOS) projected on the first surface atoms for the SIESTA calculation (dashed line) and the extended Hückel theory (EHT) fit (solid lines) for (a) the $p(2$ $\times 2)_{\mathrm{O} \text { fcc }+\mathrm{CO} \text { top }}$ model of Fig. 12(c), (b) the clean Ir tip, and (c) the oxygen terminated Ir-O tip. The insets at the top of the figures give side and normal views of each system. The solid thick lines in the normal views indicate the unit cell. For the tips, although the SIESTA calculations are performed in a $p(3 \times 3)$ lattice, the STM simulations assume an isolated apex, as indicated in the figures. The examples show the level of agreement achieved for the structures considered in this work.

consistent DFT Hamiltonian by the EHT. Within the EHT, each AO is described in terms of one or two Slater orbitals; in this work we defined a reduced $s p d \mathrm{AO}$ basis for Ir and $\mathrm{Pd}$ and a $s p$ basis for $\mathrm{O}$ and C. Despite its simplicity, it has been shown that, after a proper parametrization, the EHT can provide an accurate description of the electronic structure of bulk systems. ${ }^{27}$ The parametrization scheme consists of a least squares fit of the EHT band structure to the DFT derived band structure, using as parameters the $\mathrm{AO}$ on-site energies and their Slater exponents and coefficients. In this work, we first parametrized the AO basis for bulk Ir and bulk Pd following the above scheme. Next, similar fits were carried out for all calculated slabs (both surfaces and tips). In order to speed up the fit and reduce the number of parameters, those atoms with a similar chemical environment were assigned the same EHT parameters, while only the AO onsite energies were varied. Furthermore, since the band structure does not contain any atom specific information, the least squares fits also included the density of states projected onto each atom of the slab (PDOS) in order to ensure that a correct fit is achieved atom by atom.

Figure 1(a) shows an example for such a fit for the $p(2$ $\times 2)+\mathrm{O}_{\text {fcc }}+\mathrm{CO}_{\text {top }}$ model of Fig. 12(c) (described in Sec. V). The DOS projected on the first surface atoms is shown for 
the siesta calculation (dashed line) and the EHT fit (solid lines). The Pd $d$-band position and width are accurately reproduced, both for the $\mathrm{Pd}$ below the $\mathrm{CO}\left(\mathrm{Pd}-1_{\mathrm{CO}}\right)$ and for the Pd's bonded to the $\mathrm{O}\left(\mathrm{Pd}-1_{\mathrm{O}}\right)$. All peaks relevant to the adsorbates, O and CO, are also correctly fitted. Similar levels of agreement were achieved for all other surface slabs considered in this work, signaling a good transferability of the EHT AO basis. In Figs. 1(b) and 1(c) we show the fits for the Ir and IrO tips. Again, a very good correspondence is obtained between the DFT results and the EHT parametrization.

\section{STM simulations}

The GREEN code was employed to simulate the STM images. Both the tip and the sample were modeled as semiinfinite materials, each with its own 2D lattice except for the tip apex, which was considered as isolated. The entire STM system was then described atom by atom, employing the DFT optimized geometries for the sample surface and the tip apex. The electronic tunneling current was evaluated assuming zero temperature

$$
I(V)=\frac{e}{\pi \hbar} \int_{-e V / 2}^{+e V / 2} d E T_{T S}(E, V)
$$

where the transmission coefficient $T_{T S}(E, V)$, giving the probability of an electron tunneling from the substrate to the tip, is evaluated within the elastic approximation ${ }^{36}$ and assuming that the potential drop entirely occurs in the STM vacuum region

$$
\begin{aligned}
T_{T S}(E, V)= & \operatorname{Tr}\left[G_{T S}\left(E_{F}^{m}+E\right) \Gamma_{s}\left(E_{F}^{s}-e V / 2+E\right)\right. \\
& \left.\times G_{T S}^{\dagger}\left(E_{F}^{m}+E\right) \Gamma_{t}\left(E_{F}^{t}-e V / 2+E\right)\right],
\end{aligned}
$$

where $E_{F}^{t}$ and $E_{F}^{s}$ refer to the Fermi levels at the tip and sample, respectively, and $E_{F}^{m}$ is their mean value. $\Gamma_{s}(E)$ and $\Gamma_{t}(E)$ give the imaginary part of the surface and tip selfenergies, respectively, while $G_{T S}(E)$ is the Green's function linking the surface to the tip. Given the small tip-sample coupling $H_{T S}$ we evaluate $G_{T S}(E)$ only up to first order in $H_{T S}$. This approach is equivalent to neglecting second order or higher tunneling events.

The different 2D periodicities and possible symmetries present in each part of the system were fully taken into account by means of an efficient $k$-sampling scheme, ${ }^{30}$ we employed well over $200 k$ points for the bulk tip and bulk sample layers. The imaginary part of the energy $\epsilon_{i}$ was set to $10 \mathrm{meV}$ for the sample and tip bulk regions, while at the surface layer and the tip apex it was increased to $50 \mathrm{meV}$. The energy integration step used to numerically integrate Eq. (1) was accordingly set to $50 \mathrm{meV}$.

In order to simulate topographic images, the tip-surface distance was iteratively varied at each pixel in the image until the desired current value is achieved. Rarely, variations in the appearance of the images were detected depending on the relative orientation of the tip with respect to the sample. Hence, a rotation of the entire tip block about the $z$ axis was included as a further parameter when comparing the experimental images to the simulated ones.
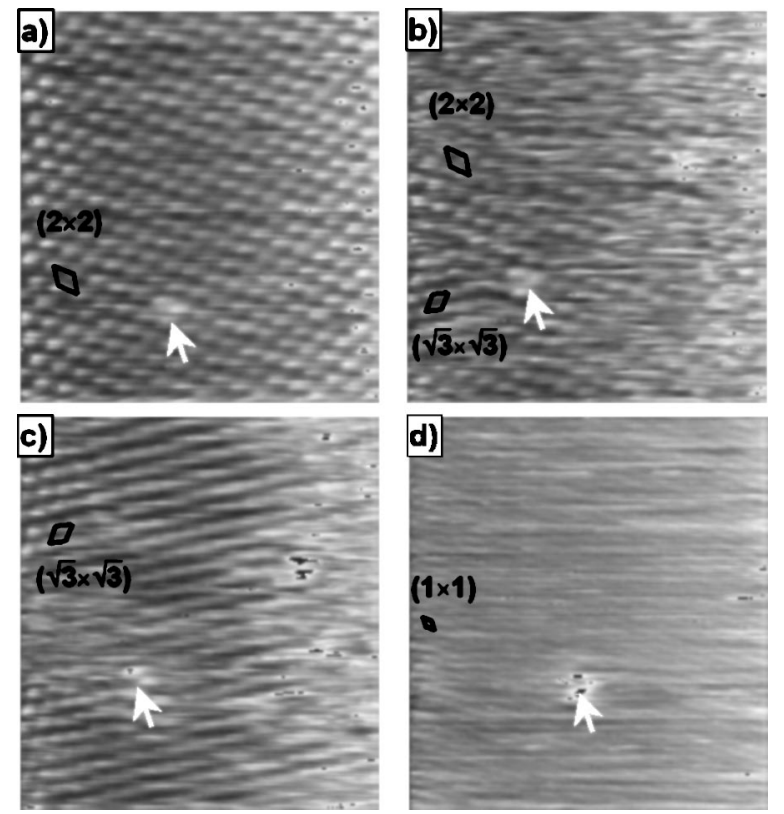

FIG. 2. Four STM images $(9 \mathrm{~nm} \times 9.5 \mathrm{~nm})$ from a series recorded with the video-STM at $300 \mathrm{~K}$, the $\mathrm{CO}$ pressure was 1 $\times 10^{-7}$ Torr. (a) Initial surface covered with the $p(2 \times 2)_{\mathrm{O}}$ structure $(t=0 \mathrm{~s}),(\mathrm{b})$ the $(\sqrt{3} \times \sqrt{3}) R 30_{\mathrm{O}}^{\circ}$ structure starts to form at the lowerleft of the image $(t=20 \mathrm{~s})$, (c) complete $(\sqrt{3} \times \sqrt{3}) R 30_{\mathrm{O}}^{\circ}$ structure; the local $\mathrm{O}$ coverage has changed from $\theta=1 / 4$ to $\theta=1 / 3(t=22 \mathrm{~s})$, (d) final stage, with the surface completely covered by $\mathrm{CO}$. The $p(1 \times 1)$ periodicity of the palladium substrate is weakly resolved $(t=48 \mathrm{~s})$. The frames are from the same area, see arrow pointing to the same defect. Tunnel parameters are $V=+0.32 \mathrm{~V}, I=1.4 \mathrm{nA}$.

\section{RESULTS}

STM images obtained with clean metallic tips typically show oxygen atoms as dark ${ }^{37}$ and $\mathrm{CO}$ as bright features. ${ }^{20}$ With adsorbed foreign atoms at the tip, the contrast may change. For example, with oxygen at the tip apex the contrast inverts and oxygen appears bright. ${ }^{38-40}$ During gas adsorption experiments, the tip often changed, and STM images presented here correspond to both situations. We denote as "normal contrast" that of the clean tip and as "reversed contrast" that of a possibly oxygen terminated tip. Despite these changes of the imaging conditions the development of the structures usually allowed us to correlate the observed surface areas with the respective adsorbates.

\section{A. Phase transitions at room temperature}

In the first set of experiments the $p(2 \times 2)$ oxygencovered surface was exposed to $\mathrm{CO}$ at room temperature, the structure changes were monitored by video-STM. Figure 2 shows four STM images extracted from a longer video sequence obtained during exposure to $\mathrm{CO}$. The image acquisition rate was 20 images/s and the entire process is complete within $50 \mathrm{~s}$. Starting from the $p(2 \times 2)_{\mathrm{O}}$ structure at $t=0$ [Fig. 2(a)], adsorption of $\mathrm{CO}$ induces the formation of a $(\sqrt{3}$ $\times \sqrt{3}) R 30_{\mathrm{O}}^{\circ}$ structure. During several images the structure on the scanned areas fluctuates between the $p(2 \times 2)_{\mathrm{O}}$ and $(\sqrt{3}$ $\times \sqrt{3}) R 30_{\mathrm{O}}^{\circ}$ phases. In Fig. 2(b) a small area of $(\sqrt{3}$ 

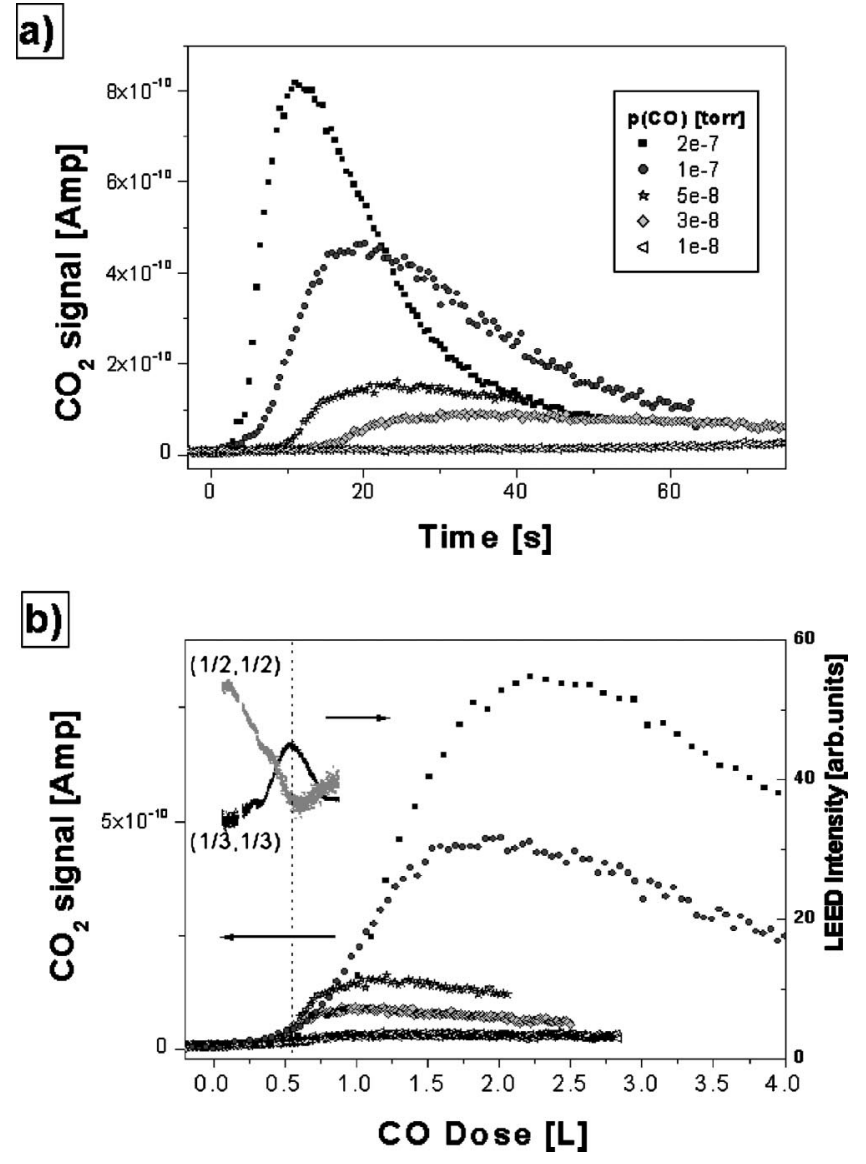

FIG. 3. Mass spectrometer data showing the $\mathrm{CO}_{2}$ production rate for different $\mathrm{CO}$ pressures between $5 \times 10^{-9}$ and 5 $\times 10^{-7}$ Torr. (a) Plotted as a function of time. The integral of the curves are constant, as is expected for a fixed oxygen coverage $(\sim 0.25 \mathrm{ML})$. (b) Plotted as a function of $\mathrm{CO}$ dose in $\mathrm{L}(1 \mathrm{~L}$ $=10^{-6}$ Torr $\left.\mathrm{s}\right)$. The inset shows LEED intensities for the $(1 / 3,1 / 3)$ and $(1 / 2,1 / 2)$ spots at $9.9 \times 10^{-9}$ Torr CO. Note the induction phase for $\mathrm{CO}_{2}$ production, where the $\mathrm{CO}_{2}$ signal stays low. After a $\mathrm{CO}$ exposure of $\sim 0.55 \mathrm{~L}$ the rate increases rapidly. This point also corresponds to a maximum of the $(1 / 3,1 / 3)$ and a minimum of the $(1 / 2,1 / 2)$ LEED spot intensities.

$\times \sqrt{3}) R 30_{\mathrm{O}}^{\circ}$ can be seen at the lower left part of the image. A few seconds later this structure covers the entire scanning area [Fig. 2(c)]. Subsequently, the $(\sqrt{3} \times \sqrt{3}) R 30_{\mathrm{O}}^{\circ}$ structure disappears and the surface enters a disordered stage. Finally, in Fig. 2(d), where the surface is covered by CO, only the $(1 \times 1)$ periodicity of the palladium substrate is resolved. At room temperature the $\mathrm{CO}$ molecules are too mobile to resolve ordered arrangements; only in the surroundings of defects some weak periodicities were observed.

The analogous experiment in front of the LEED optics shows an evolution of LEED patterns [inset of Fig. 3(b)] similar to those described by Conrad et al. ${ }^{11}$ The $p(2 \times 2)$ pattern changes into a $(\sqrt{3} \times \sqrt{3}) R 30^{\circ}$ pattern, which disappears after further $\mathrm{CO}$ adsorption.

The mass spectrometer data allow to correlate the observed structures with the reaction to give $\mathrm{CO}_{2}$. The plots of the $\mathrm{CO}_{2}$ formation rate as a function of time [Fig. 3(a)] show a constant integral. The area under the curves is proportional to the amount of $\mathrm{CO}_{2}$ produced and thus also to the amount of adsorbed $\mathrm{O}$. That the area is constant evidences that the measured $\mathrm{CO}_{2}$ mainly comes from the sample and spurious $\mathrm{CO}_{2}$ from the chamber walls can be neglected. The curves further show that the reaction sets in only after a certain time delay, which increases with decreasing $\mathrm{CO}$ pressure. When the same data are plotted as functions of the $\mathrm{CO}$ coverages [Fig. 3(b), where we assume a sticking coefficient of 1, and therefore, $1 \mathrm{~L}=10^{-6}$ Torr s], it turns out that this onset always happens at the same exposure, approximately $0.5 \mathrm{~L}$, independently of the $\mathrm{CO}$ pressure. One can conclude that there is an induction period that only depends on the amount of $\mathrm{CO}$ dosed. After this induction, the rate increases rapidly.

The evolution of the LEED pattern (at $9.9 \times 10^{-9}$ Torr $\mathrm{CO}$ ) is also plotted in Fig. 3(b) (inset). It is seen that the maximum of the $(1 / 3,1 / 3)$ spot intensity occurs at $0.55 \mathrm{~L}$, pretty much exactly at the end of the induction phase of the reaction (dashed line). The precise exposure, at which the maximum occurs, only varies between 0.5 and $0.6 \mathrm{~L}$ for $\mathrm{CO}$ pressures between $7.5 \times 10^{-9}$ Torr and $7.5 \times 10^{-8}$ Torr; at other pressure ranges there is a larger error in the determination of the maximum because of very short times or of weak spot intensities. The maximum in the $(1 / 3,1 / 3)$ intensity coincides with the minimum in the $(1 / 2,1 / 2)$ spot intensity. These data strongly support the assumption, and this has not been directly demonstrated before, that the structures formed on this surface are indeed important for the reaction.

\section{B. Phase transitions at low temperatures}

To better resolve the evolution of the structures during the $\mathrm{CO}$-induced compression and reaction of the $\mathrm{O}$ layer, the temperature was lowered, and the experiments were performed by means of a variable temperature STM. In order to relate our findings with the $200 \mathrm{~K}$ LEED investigations by Conrad et al., ${ }^{11}$ the investigations were performed in the same temperature range (170 to $190 \mathrm{~K})$. Figure 4 shows characteristic STM images from different stages of the reaction. The images do not correspond to the same scanning area, because thermal drift was intentionally not compensated to reduce tip-shadowing effects.

The initial stage shows the oxygen $(2 \times 2)$ structure [Fig. 4(a)], which is similar to that of Fig. 2(a), but has some vacancies. In this case, the oxygen atoms are imaged bright, corresponding to a "reversed-contrast" situation. After starting the $\mathrm{CO}$ exposure, a slight improvement of the $p(2$ $\times 2)_{\mathrm{O}}$ structure is observed (not shown here). Then, domain boundaries occur, and bright features form at the domain boundaries [Fig. 4(b)]. After further $\mathrm{CO}$ dosing, dark areas (probably oxygen free) appear, as observed in Fig. 4(c), while the rest of the surface still has the $(2 \times 2)_{\mathrm{O}}$ phase. The following image [Fig. 4(d)] shows that the $p(2 \times 2)_{\mathrm{O}}$ phase is transformed into a $(\sqrt{3} \times \sqrt{3}) R 30^{\circ}$ structure. This new ordered phase is interpreted as consisted of oxygen, i.e., $(\sqrt{3}$ $\times \sqrt{3}) R 30_{\mathrm{O}}^{\circ}$, because its formation from the $p(2 \times 2)_{\mathrm{O}}$ structure could be followed. The next step is the formation of a $p(2 \times 1)$ phase in the oxygen areas, which is characterized by a striped structure [Fig. 4(e)]. There are rotational domains oriented in the three equivalent directions of the substrate. At 

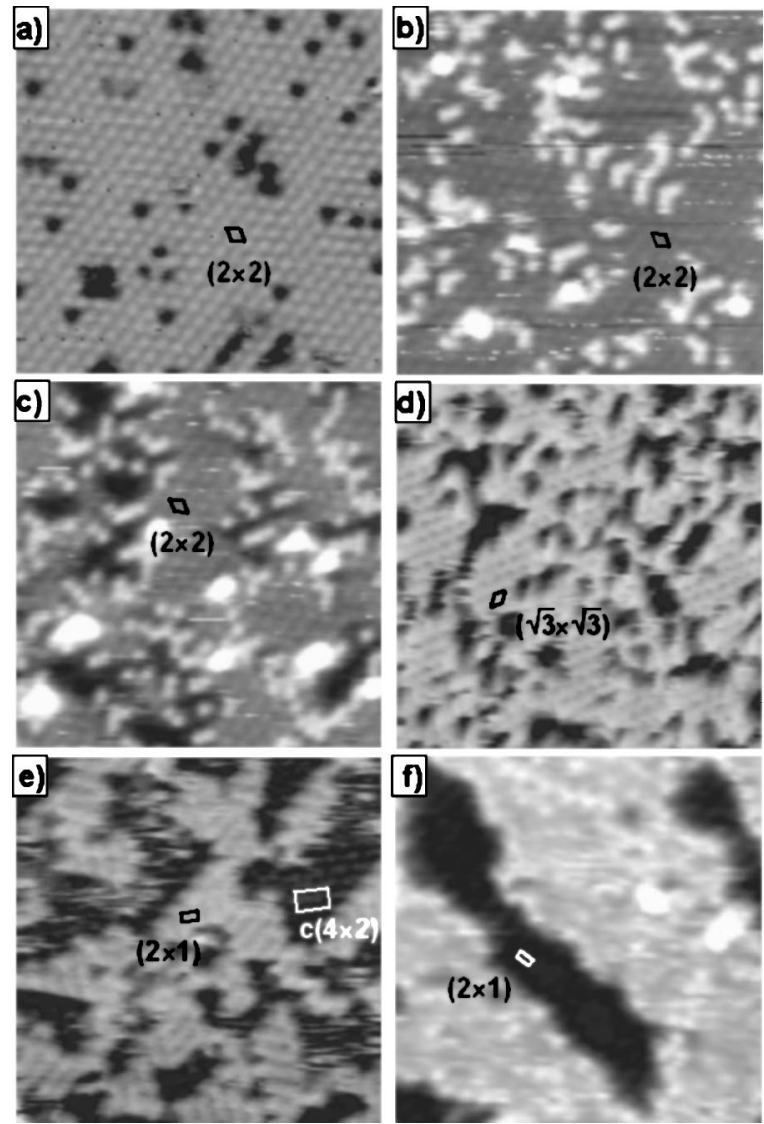

FIG. 4. STM images $(12 \mathrm{~nm} \times 12 \mathrm{~nm})$ recorded during adsorption of $\mathrm{CO}$ at $170-190 \mathrm{~K}$. (a) Oxygen $p(2 \times 2)_{\mathrm{O}}$ phase; $V=-0.4 \mathrm{~V}$, $I=1 \mathrm{nA}, T=170 \mathrm{~K}$. (b) $p(2 \times 2)_{\mathrm{O}}$ structure with bright features; $V$ $=+0.4 \mathrm{~V}, I=1 \mathrm{nA}, T=170 \mathrm{~K}$. (c) Dark areas appear; $V=+0.4 \mathrm{~V}$, $I=0.9 \mathrm{nA}, T=173 \mathrm{~K}$. (d) $(\sqrt{3} \times \sqrt{3}) R 30_{\mathrm{O}}^{\circ}$ structure; $V=-1.1 \mathrm{~V}, I$ $=0.7 \mathrm{nA}, T=181 \mathrm{~K}$. (e) $p(2 \times 1)_{\mathrm{O}}$ and $\mathrm{c}(4 \times 2)_{\mathrm{CO}}$ phases; $V$ $=1.75 \mathrm{~V}, I=0.66 \mathrm{nA}, T=184 \mathrm{~K}$. (f) $p(2 \times 1)_{\mathrm{O}}$ islands and $\mathrm{CO}$ covered surface; $V=-1.7 \mathrm{~V}, I=0.75 \mathrm{nA}, T=176 \mathrm{~K}$. Images (a)-(e) correspond to "reversed-contrast" tip conditions, with oxygen imaged as bright bumps. (f) corresponds to "normal-contrast" with oxygen imaged dark.

the same time, the dark areas transform into structures that can be associated with the $\mathrm{CO}$ molecules [gray areas in Fig. 4(e)]. These areas briefly show a $(\sqrt{3} \times \sqrt{3}) R 30^{\circ}$ periodicity [caused by the $(\sqrt{3} \times \sqrt{3}) R 30^{\circ}$ CO phase, not shown here] and then a phase with a rectangular structure, which is interpreted as the $c(4 \times 2)_{\mathrm{CO}}$ phase. Both $\mathrm{CO}$ structures have been observed before by STM by Rose et al. ${ }^{20}$ In this stage [Fig. $4(\mathrm{e})]$, the island shapes and contours change quickly. The horizontal lines [right-lower corner in Fig. 4(e)] reveal site exchanges on the time scale of the lines of the STM scan, reflecting a significant mobility of the adsorbates. The $p(2$ $\times 1)_{\mathrm{O}}$ islands change their shapes from more-or-less triangular in Fig. 4(e) to elongated bands in Fig. 4(f). The elongation of the $p(2 \times 1)$ oxygen areas is perpendicular to the direction of the stripes inside the island. In Fig. 4(f) most of the surface is covered with $\mathrm{CO}$ (bright) with unresolved, but more complex structures (see also Ref. 20). Because the $p(2 \times 1)_{\mathrm{O}}$ islands appear darker than the surrounding $\mathrm{CO}$ phase this last STM image is interpreted as a "normal-
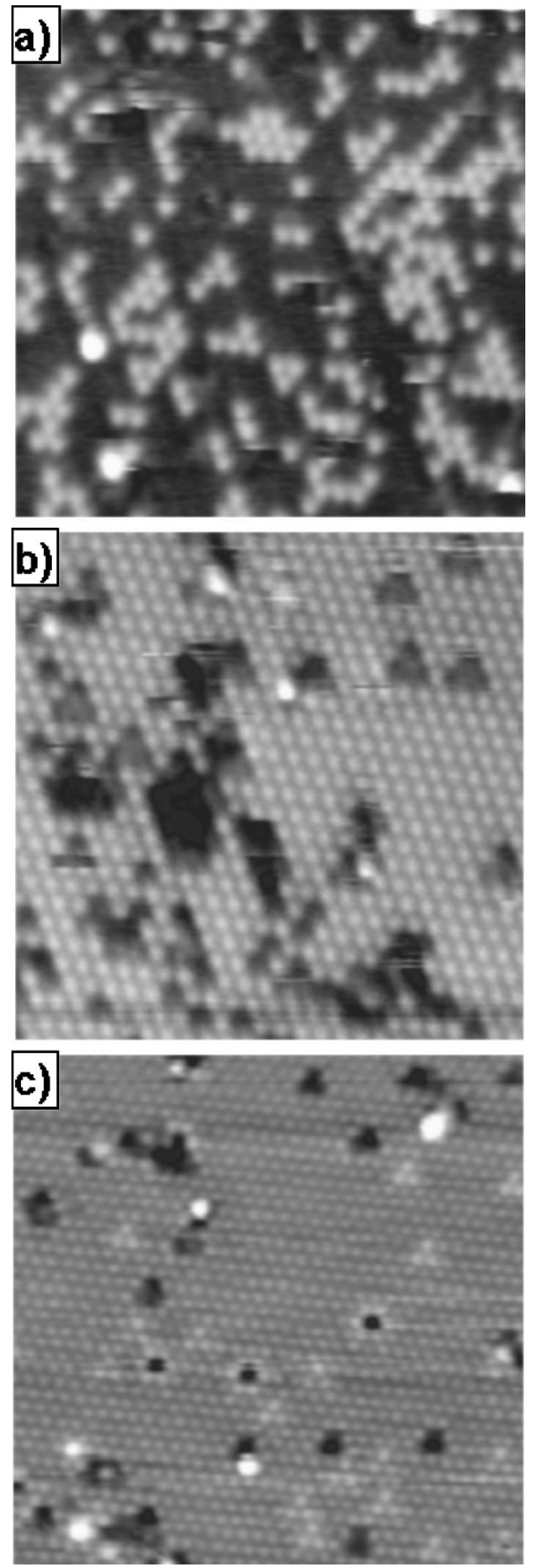

FIG. 5. Formation of the $p(2 \times 2)_{\mathrm{CO}+\mathrm{O}}$ mixed structure. (a) STM image $(15.5 \mathrm{~nm} \times 15.5 \mathrm{~nm})$ recorded at $60 \mathrm{~K}$ showing $\mathrm{CO}$ molecules starting to form a $(2 \times 2)$ structure of bright features. The $\mathrm{CO}$ dose is $2 \mathrm{~L}$. $V=-0.85 \mathrm{~V}, \quad I=0.9 \mathrm{nA}$. (b) Image $(13.4 \mathrm{~nm}$ $\times 13.4 \mathrm{~nm}$ ) after $8 \mathrm{~L} \mathrm{CO}$; $V=-1.4 \mathrm{~V}, I=0.9 \mathrm{nA}, T=90 \mathrm{~K}$. (c) STM image $(18.4 \mathrm{~nm} \times 18.4 \mathrm{~nm})$ at $102 \mathrm{~K}$, with the $p(2 \times 2)_{\mathrm{CO}+\mathrm{O}}$ almost completed. $V=-1.7 \mathrm{~V}, I=0.9 \mathrm{nA}$.

contrast" image. Obviously a tip change occurred between this and the previous image.

The $p(2 \times 1)_{\mathrm{O}}$ structure was observed to be reactive, and the $p(2 \times 1)$ islands shrink as result of the reaction with the $\mathrm{CO}$ molecules. These results will be presented in another paper. $^{41}$ 
In the final stage of this experiment $\mathrm{CO}$ structures cover most of the surface, and there are few residual $p(2 \times 1)_{\mathrm{O}}$ areas. The LEED in this stage shows an invariable $c(4 \times 2)$ pattern, TDS indicates that the surface after the STM experiments is mainly $\mathrm{CO}$ covered and that most of the oxygen has been reacted off, but still there is some oxygen left.

\section{Coadsorption at temperatures below $110 \mathrm{~K}$}

To suppress the structure rearrangements at 170-190 K, $\mathrm{CO}$ adsorption experiments were also performed between 110 and $60 \mathrm{~K}$, the lowest temperature we can currently reach with our variable-temperature STM. Under these conditions the oxygen atoms are immobile, and it is expected that the compression into the $(\sqrt{3} \times \sqrt{3}) R 30_{\mathrm{O}}^{\circ}$ structure no longer occurs. Figure 5 shows STM images corresponding to three stages of the $\mathrm{CO}$ adsorption on the $p(2 \times 2)_{\mathrm{O}}$-precovered surface in this temperature range. Figure $5(\mathrm{a})$, obtained after adsorption of $2 \mathrm{~L}$ of $\mathrm{CO}$ at $60 \mathrm{~K}$, shows several bright features forming local $p(2 \times 2)$ periodicities. The number of bright features grows with $\mathrm{CO}$ exposure, evidencing that they are $\mathrm{CO}$ molecules adsorbed between the $\mathrm{O}$ atoms of the $(2 \times 2)$ phase. Consecutive images (not shown here) reveal several hopping events of $\mathrm{CO}$ between neighboring $p(2$ $\times 2$ ) sites, so that the $\mathrm{CO}$ molecules are still mobile between the oxygen atoms at this low temperature. In Fig. 5(b) the bright $p(2 \times 2)$ structure is almost complete. The horizontal lines mark movement of $\mathrm{CO}$ molecules at the boundaries of the $p(2 \times 2)$ structure. By slightly increasing the temperature up to $100 \mathrm{~K}$ the entire area became covered with this $p(2$ $\times 2)$ structure [Fig. 5(c)]. At higher temperatures the $\mathrm{CO}$ molecules are able to diffuse into the scanning area from outside. A detail of this phase is shown in Fig. 6, which also displays triangular and hexagonal point defects; these are most likely caused by foreign atoms at different adsorption sites. Since $\mathrm{CO}$ alone does not form a $p(2 \times 2)$ structure at these low coverages [a $p(2 \times 2) \mathrm{CO}$ structure exists for $\theta$ $=3 / 4$ (Refs. 17 and 20)] and because oxygen is present on the surface, it can be concluded that the structure represents

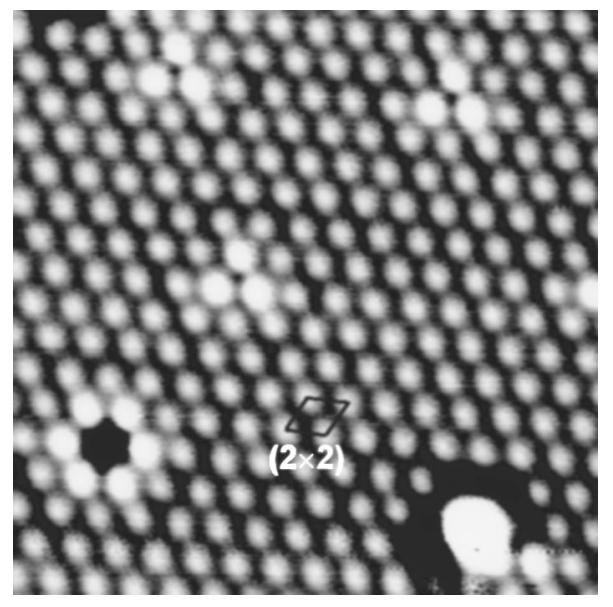

FIG. 6. STM image $(8.1 \mathrm{~nm} \times 8.5 \mathrm{~nm})$ with the completed $p(2$ $\times 2)_{\mathrm{CO}+\mathrm{O}}$. $\mathrm{CO}$ molecules around defects appear brighter. $V=-1.7 \mathrm{~V}, I=0.9 \mathrm{nA}, T=103 \mathrm{~K}$. a mixed $p(2 \times 2)_{\mathrm{O}+\mathrm{CO}}$ phase. The corrugation of this phase is $0.25 \AA$, higher than of the previous phases.

LEED after the STM experiments showed a sharp $p(2$ $\times 2)$ pattern. In the TDS desorption peaks for $\mathrm{CO}$, oxygen and $\mathrm{CO}_{2}$ were detected (the latter produced during the TDS), again demonstrating that $\mathrm{O}$ and $\mathrm{CO}$ coexist in this phase.

\section{STRUCTURES: COMPARISON BETWEEN EXPERIMENT AND THEORY}

In this section the different structures observed by STM between 60 and $300 \mathrm{~K}$ are investigated in detail. We compare the images with STM simulations performed for a wide variety of models.

\section{A. The oxygen $p(2 \times 2)$ structure}

After adsorption of oxygen at room temperature, the $\operatorname{Pd}(111)$ surface shows a $p(2 \times 2)_{\mathrm{O}}$ LEED pattern, as was reported before. ${ }^{7}$ We find that the quality of the pattern can be improved (i) by adsorbing oxygen at $230 \mathrm{~K}$ or (ii) by adsorbing molecular oxygen at $170 \mathrm{~K}$, followed by annealing over $200 \mathrm{~K}$. This temperature of $200 \mathrm{~K}$ is the lowest limit to form the $p(2 \times 2)_{\mathrm{O}}$ structure, as it is the temperature to complete the dissociation of the oxygen molecules. ${ }^{42}$ Below this temperature, the oxygen is adsorbed as molecules.

STM images of the oxygen covered surface at room temperature show a $p(2 \times 2)$ structure, and reveal fast diffusion of the oxygen atoms over the Pd surface. Even at the high scanning rates of 20 images per second, which can be achieved with the video STM, we have not been able to

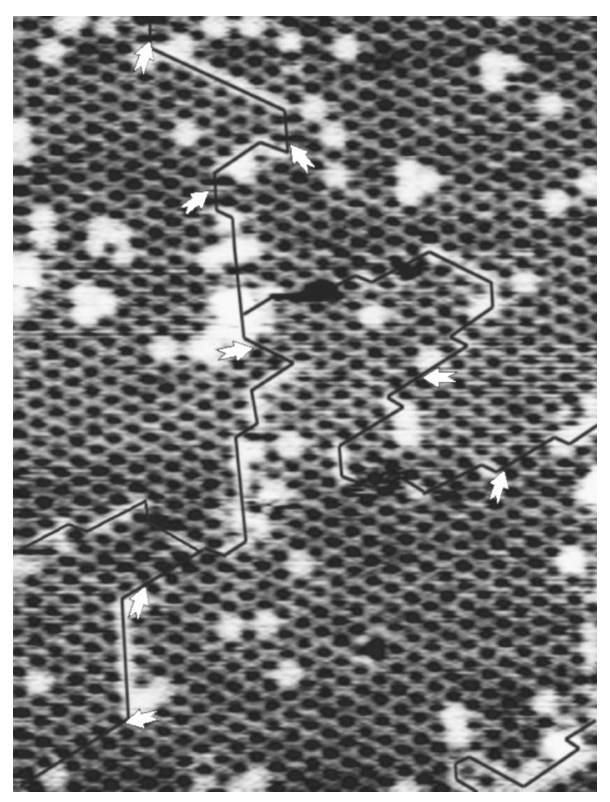

FIG. 7. STM image $(15.4 \mathrm{~nm} \times 21.8 \mathrm{~nm})$ recorded at $176 \mathrm{~K}$ of the oxygen covered $\mathrm{Pd}(111)$ surface showing the $p(2 \times 2)_{\mathrm{O}}$ structure. At this temperature the oxygen atoms (dark dots, i.e., "normal contrast") are almost immobile, so that the structure and the vacancies (bright) are well resolved. At the domain boundaries, indicated by the line, some oxygen atoms occupy $\sqrt{3}$ positions (marked with arrows). $V=-1.4 \mathrm{~V}, I=1.05 \mathrm{nA}$. 

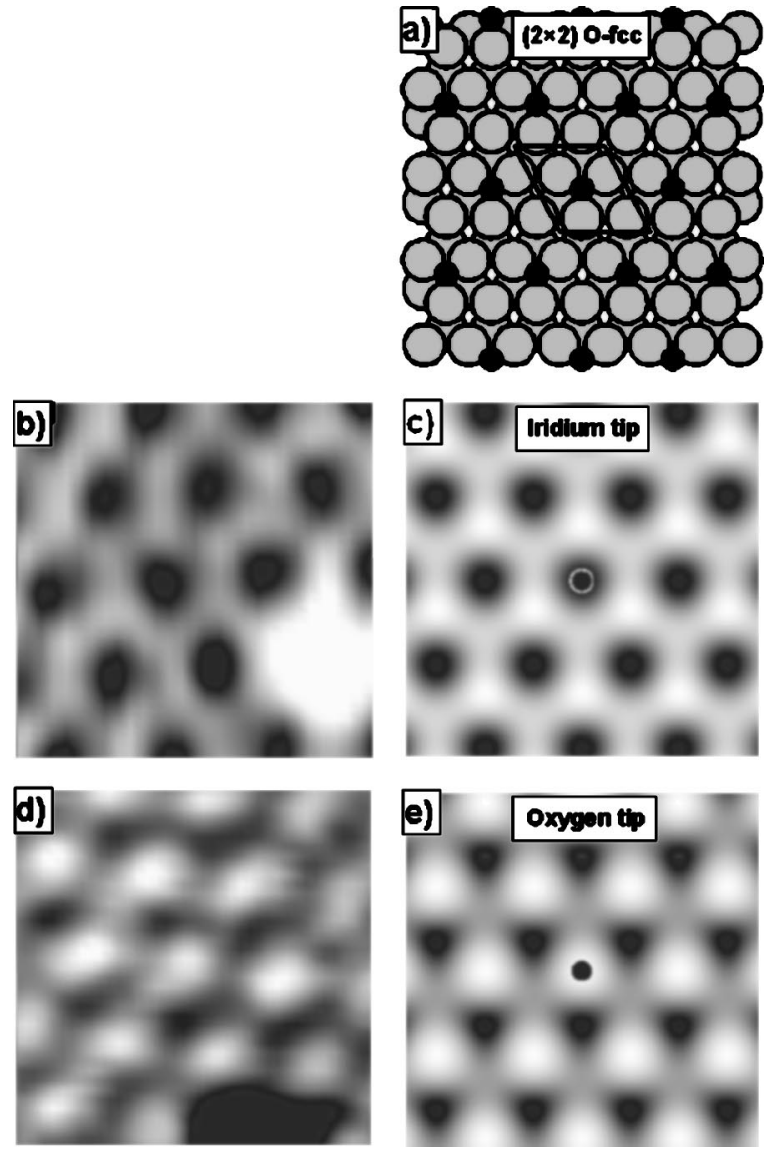

FIG. 8. Comparison between experimental and simulated STM images for the oxygen $p(2 \times 2)_{\mathrm{O}}$ structure on $\mathrm{Pd}(111)$. (a) Model with oxygen on the fcc position. (b) STM image $(2 \mathrm{~nm} \times 2 \mathrm{~nm})$ obtained with normal contrast showing oxygen atoms as dark and vacancies as bright features; $V=-1.4 \mathrm{~V}, I=1.05 \mathrm{nA}$. (c) Simulated image calculated for the same tunnel conditions as in (b) but with the iridium tip depicted in Fig. 1(b). (d) STM image $(2 \mathrm{~nm}$ $\times 2 \mathrm{~nm}$ ) obtained with inverted contrast, where oxygen atoms appear bright; $V=-0.4 \mathrm{~V}, I=1 \mathrm{nA}$. (e) Simulated image at the same tunnel conditions as in (c) for an oxygen terminated iridium tip [depicted in Fig. 1(c)].

follow the movement of the oxygen atoms or of the vacancies in the $p(2 \times 2)_{\mathrm{O}}$ phase. However, at lower temperatures the diffusion could be sufficiently reduced to also resolve defects in the ordered structure.

Figure 7 shows an image obtained at $176 \mathrm{~K}$, where very few jumps are observed between consecutive images. At this temperature the $p(2 \times 2)_{\mathrm{O}}$ structure is obviously almost frozen in. That in this case dark dots form a hexagonal structure, similar to the $\mathrm{O}$ atoms in the $(2 \times 2)_{\mathrm{O}}$ phase, indicates that the oxygen atoms are imaged dark here ("normal contrast"). The bright sites can accordingly be interpreted as vacancies. Dark oxygen features have similarly been reported for other metal surfaces such as $\mathrm{Pt}^{37,43}$ Domain boundaries are clearly visible at this temperature (marked in the figure with black lines). Directly at the domain boundaries, some oxygen atoms appear at smaller interatomic separations corresponding to $\sqrt{3}$ distances (arrows).

Figure 8 compares the experimental STM images of the $p(2 \times 2)_{\mathrm{O}}$ structure and simulated images for both the clean Ir and the oxygen terminated tips. As determined by DFT calculations, ${ }^{21,44}$ the oxygen atoms are adsorbed at threefold fcc sites [see model in Fig. 8(a)]. The result of the STM simulations [Fig. 8(c)] is that for clean iridium tips the tunnel current is reduced over the oxygen atoms of the $p(2 \times 2)_{\mathrm{O}}$, so that the oxygen atoms are imaged as dark features by the STM [Fig. 8(b)]. For oxygen-terminated tips, the contrast is inverted, and the oxygen atoms appear as protrusion [Fig. $8(e)]$ which can be directly correlated with an inverted contrast STM image shown in Fig. 8(d). This effect has been analyzed in detail by other authors. ${ }^{38,39}$ For the tunnel conditions used in the experiments shown in Figs. 7 and 8(b), for a clean iridium tip, the oxygen atoms appear circular. Different tunnel conditions produce the oxygen atoms to appear triangular, with hcp or top as brightest positions depending on the tunnel bias voltage. ${ }^{39}$

\section{B. The oxygen $(\sqrt{3} \times \sqrt{3}) R 30^{\circ}$ structure}

The STM data (Fig. 4) clearly show that CO adsorption on the $p(2 \times 2)_{\mathrm{O}}$ structure causes compression of the $\mathrm{O}$ over-
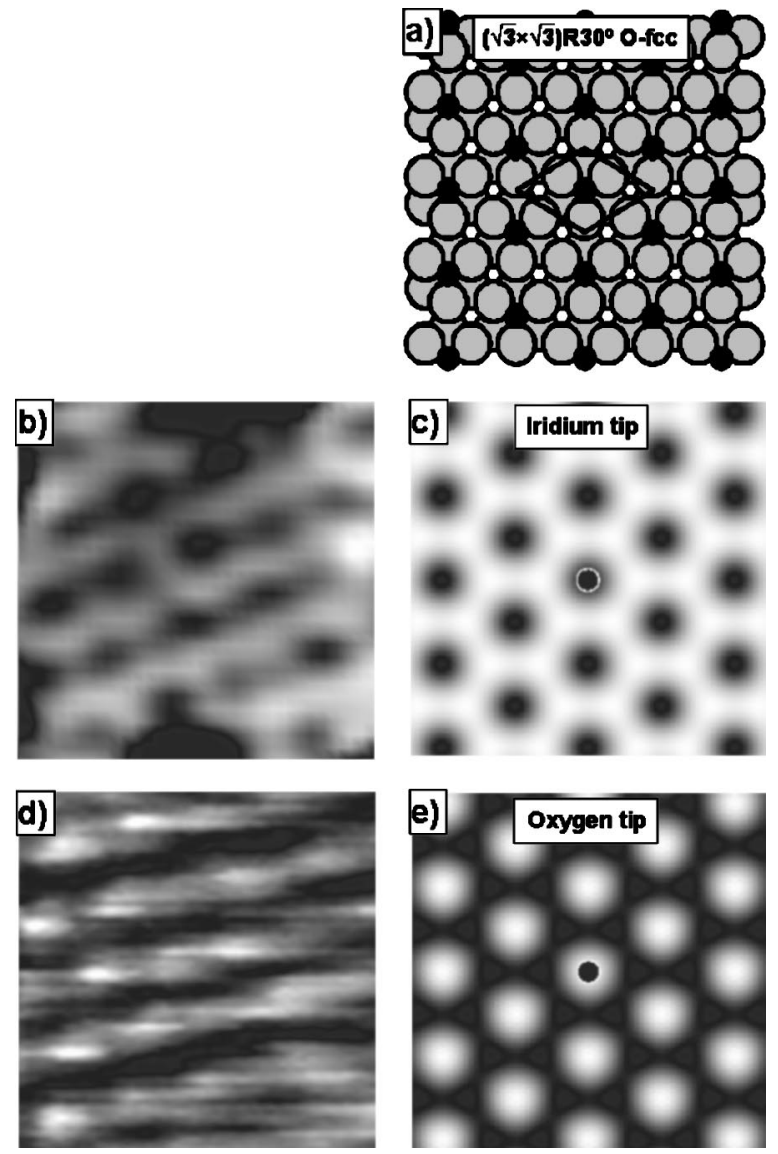

FIG. 9. The $(\sqrt{3} \times \sqrt{3}) R 30^{\circ}$ oxygen structure. (a) Model with oxygen atoms on threefold fcc positions. (b) STM image $(2 \mathrm{~nm}$ $\times 2 \mathrm{~nm}) ; T=181 \mathrm{~K}, V=-1.1 \mathrm{~V}, I=0.7 \mathrm{nA}$; corrugation is $0.3 \AA$. (c) Simulation with an $\operatorname{Ir}(111)$ tip, in which the oxygen atoms appear dark. (d) STM image $(2 \mathrm{~nm} \times 2 \mathrm{~nm}) ; V=+0.32 \mathrm{~V}, I=1.4 \mathrm{nA}$. (e) Simulation with an oxygen terminated tip where the oxygen atoms appear bright. 
layer into domains of a $(\sqrt{3} \times \sqrt{3}) R 30^{\circ}$ structure, as had been concluded from LEED experiments. ${ }^{11,45}$ LEED-IV investigations and DFT revealed that this structure has oxygen in the fcc positions. ${ }^{21} \mathrm{~A}$ ball model of this $(\sqrt{3} \times \sqrt{3}) R 30_{\mathrm{O}}^{\circ}$ phase is sketched in Fig. 9(a). Similarly as the $(2 \times 2)_{O}$ structure this phase was either imaged as a hexagonal arrangement of dark features [Fig. 9(b)], or of bright features [Fig. 9(d)]. In analogy with the $(2 \times 2)_{\mathrm{O}}$ phase, these effects reflect tip changes, i.e., normal contrast or inverted contrast, and can be correlated with the calculated STM images for an iridium tip [Fig. 9(c)] and for an oxygen terminated tip [Fig. 9(e)]. The comparison shows a good qualitative agreement with the simulation performed. A difference between the calculated and measured image is the corrugation $(0.15$ vs. $0.30 \AA$ for the calculation and the experiment at normal contrast, respectively), despite both correspond to the same tunneling conditions. We attribute this discrepancy to the tip apex structure, which is presumably more complex in the experiment, and to possible atomic relaxations both at the tip and the substrate during the scan. ${ }^{46}$

\section{The oxygen $p(2 \times 1)$ structure}

LEED experiments have shown that further adsorption of $\mathrm{CO}$ at low temperature leads to formation of a $p(2 \times 1)$ structure. $^{11,22,45}$ In agreement with these observations, STM experiments at approximately $170 \mathrm{~K}$ show such a structure after the $p(2 \times 2)_{\mathrm{O}}$ structure. Below this temperature, the $(\sqrt{3} \times \sqrt{3}) R 30_{\mathrm{O}}^{\circ}$ is not observed, as it probably transforms quickly into the more compressed $p(2 \times 1)$ structure. At room temperature, the $p(2 \times 1)$ structure was not observed, also in agreement with LEED, ${ }^{45}$ most likely because the reaction to form $\mathrm{CO}_{2}$ is too fast.

Structure models for this $p(2 \times 1)$ phase have been conflicting. Photoelectron spectroscopy data showed energy shifts of the $\mathrm{CO}$ valence orbitals with respect to the situation before the $(2 \times 1)$ formation. It was concluded that there must be chemical interactions of the $\mathrm{CO}$ molecules with the $\mathrm{O}$ atoms, suggesting a mixed $p(2 \times 1)_{\mathrm{CO}+\mathrm{O}}$ structure with alternating lines of $\mathrm{CO}$ molecules and oxygen atoms. ${ }^{11}$ The same conclusion was drawn from the angular distribution of the $\mathrm{CO}_{2}$ molecules formed by reaction of the $(2 \times 1)$ phase. ${ }^{45}$ By contrast, DFT calculations by Seitsonen et al. ${ }^{21}$ revealed strong repulsions between $\mathrm{O}$ and $\mathrm{CO}$ in such a structure, so that separate $p(2 \times 1)_{\mathrm{CO}}$ and $p(2 \times 1)_{\mathrm{O}}$ domains would be energetically favorable.

The STM simulations allow us to discriminate between the two possibilities. Calculations were performed for two different $(2 \times 1)$ structures, one containing one $O$ atom per unit cell adsorbed on the fcc site [Fig. 10(a)], the second with an additional $\mathrm{CO}$ molecule at the fcc site between the $\mathrm{O}$ atoms [Fig. 10(b)]. That $\mathrm{CO}$ would sit on the fcc site is also expected from the preferred $\mathrm{CO}$ bonding site on $\mathrm{Pd}(111)$ in other structures. ${ }^{21}$ The results of the simulations are shown in Figs. 10(d), 10(e), 10(g), and 10(h). Comparison with the experimental images for normal-contrast and reversedcontrast tip conditions [Figs. 10(c) and 10(f), respectively] allows us to rule out the mixed model. The simulated mixed model shows features between the bright rows that were
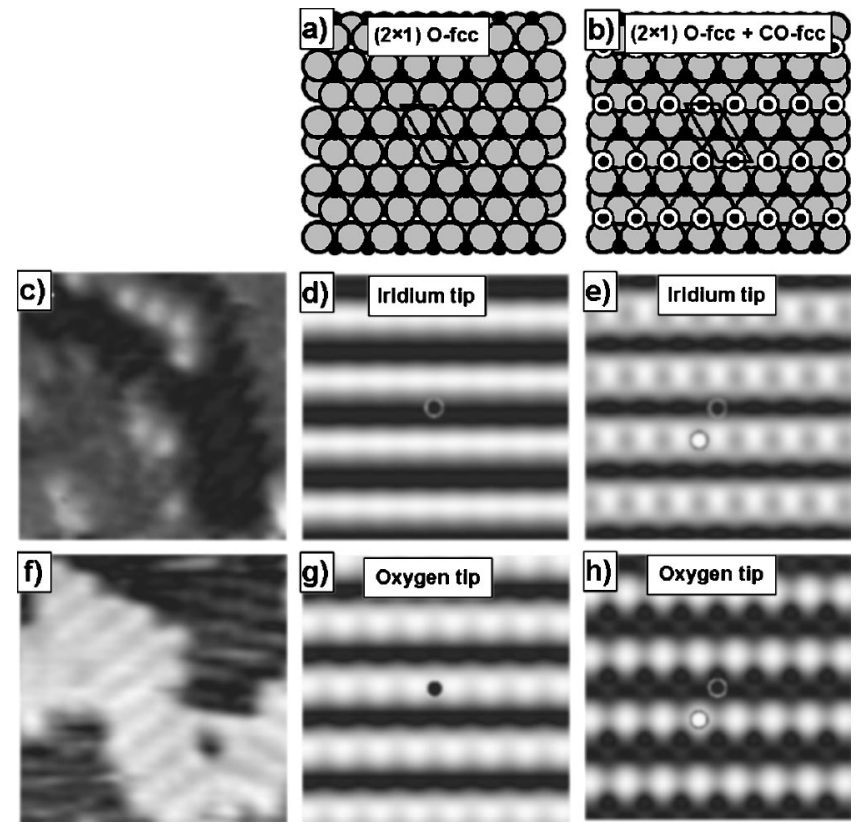

FIG. 10. The $p(2 \times 1)$ structure. (a) Model with oxygen on fcc sites. (b) Model with alternating lines of oxygen atoms and $\mathrm{CO}$ molecules. (c) STM image $(5.6 \mathrm{~nm} \times 5.6 \mathrm{~nm})$ obtained in the "normal-contrast" state; $T=63 \mathrm{~K}, V=-0.3 \mathrm{~V}, I=1 \mathrm{nA}$; corrugation is $0.1 \AA$. (d),(e) Simulated STM images performed with an iridium tip for the models in (a) and (b). (f) STM image $(4.3 \mathrm{~nm}$ $\times 4.3 \mathrm{~nm}$ ) obtained with "reversed contrast"; $V=+0.7 \mathrm{~V} ; I=1 \mathrm{nA}$; corrugation is $0.11-0.14 \AA$. (g),(h) Simulated STM images with an oxygen-terminated tip for the models in (a) and (b).

never experimentally seen, independently of the tip state. On the other hand, the pure oxygen model provides satisfactory agreement with the experiment, for both types of contrast. For a clean metal tip relatively smooth dark stripes are obtained along the $\mathrm{O}$ atom rows [Fig. 10(d)], for the oxygen terminated tip relatively smooth bright stripes [Fig. 10(g)]. Both situations occur in the experiments [Figs. 10(c) and $10(\mathrm{f})]$. We conclude that the $(2 \times 1)$ phase is a pure oxygen structure. The $\mathrm{CO}$ molecules on the areas between the $\mathrm{O}$ islands do, however, not form a $(2 \times 1)$ phase, but the known $(\sqrt{3} \times \sqrt{3}) R 30_{\mathrm{CO}}^{\circ}$ and $c(4 \times 2)_{\mathrm{CO}}$ structures. These were recently investigated by STM by Rose et al. ${ }^{20}$ their results are reproduced here for the mixed $\mathrm{O} / \mathrm{CO}$ layer.

\section{The mixed $p(2 \times 2)$ structure}

The 60-110 K STM data (Figs. 5 and 6) show that adsorption of $\mathrm{CO}$ on the $p(2 \times 2)_{\mathrm{O}}$ structure leads to formation of a new mixed $p(2 \times 2)_{\mathrm{O}+\mathrm{CO}}$ structure. At these low temperatures it can be assumed that the oxygen atoms of the $p(2 \times 2)_{\text {O }}$ phase are immobile and remain at their fcc positions. The positions of the $\mathrm{CO}$ molecules have to be determined. Figure 11(a) shows an STM image recorded at an intermediate stage of the $\mathrm{CO}$ adsorption, where only part of the $(2 \times 2)_{\mathrm{O}}$ unit cells are occupied by $\mathrm{CO}$ molecules (bright dots). Under the chosen tunnel conditions also the underlying $p(2 \times 2)_{\mathrm{O}}$ structure is weakly visible, allowing us to superimpose the $(2 \times 2)$ lattice on the image [Fig. 11(b)]. It is 

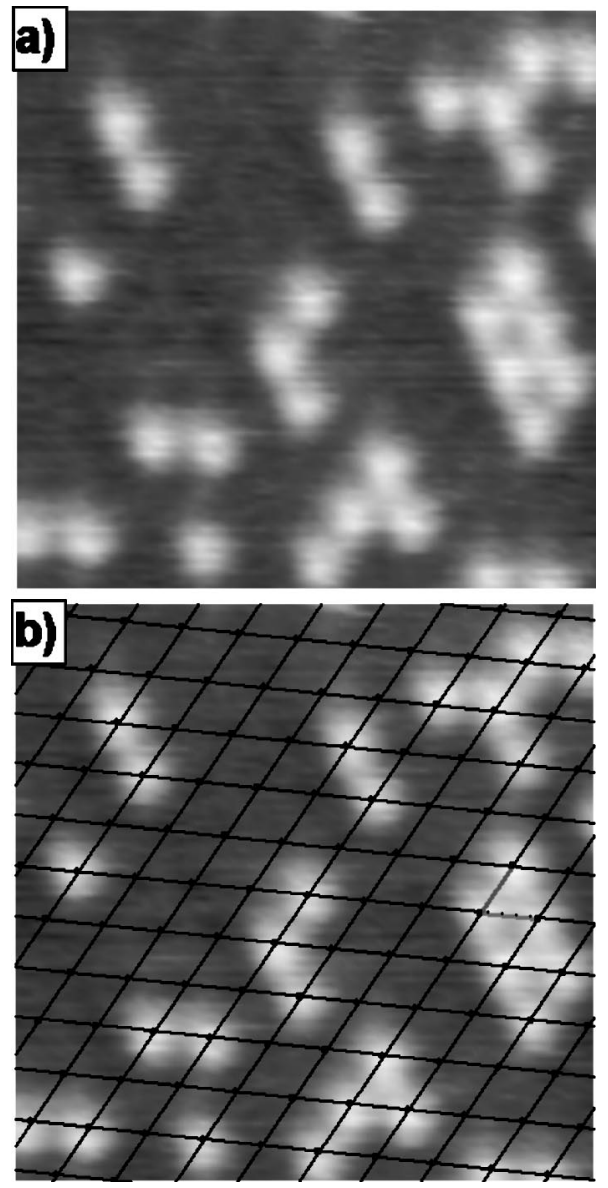

FIG. 11. (a) STM image $(3.3 \mathrm{~nm} \times 3.3 \mathrm{~nm})$ recorded during formation of the mixed $p(2 \times 2)_{\mathrm{CO}+\mathrm{O}}$ structure, $T=59 \mathrm{~K}$ and $2.5 \mathrm{~L} \mathrm{CO}$. With the applied tunneling parameters $(V=-1.4 \mathrm{~V} ; I=1.1 \mathrm{nA})$ the $\mathrm{CO}$ molecules and the underlying $p(2 \times 2)_{\mathrm{O}}$ become simultaneously visible. (b) Same image with overlaid $p(2 \times 2)$ lattice. The CO molecules are located on the same sites as the dark positions of the $p(2 \times 2)_{\mathrm{O}}$ oxygen structure. Atom-resolved images with two terraces reveal that the $\mathrm{CO}$ molecules occupy hcp sites.

found that the $\mathrm{CO}$ molecules occupy the same $(2 \times 2)$ lattice positions as the darkest sites of the $(2 \times 2)_{\mathrm{O}}$ phase. For a normal-contrast tip these would be the positions of the $\mathrm{O}$ atoms, but at $60 \mathrm{~K}$ we rule out that $\mathrm{CO}$ molecules have replaced $\mathrm{O}$ atoms. For a reversed-contrast tip the dark sites correspond to the hcp hollow sites in the centers of triplets of $\mathrm{O}$ atoms, which appears reasonable. The hcp site between three $\mathrm{O}$ atoms is therefore concluded to be the adsorption site of the $\mathrm{CO}$ molecules in the mixed $p(2 \times 2)_{\mathrm{O}+\mathrm{CO}}$ structure [Fig. 12(b)]. Other images with normal-contrast confirm this adsorption site. The possible alternative $\mathrm{CO}$ site, the on-top position in the other half of the unit cell, is therefore not in agreement with the STM data. This conclusion is supported by DFT calculations of the $p(2 \times 2)_{\mathrm{O}+\mathrm{CO}}$ structure (which had not been known experimentally at the time) that found a $0.13 \mathrm{eV}$ more stable position at the hcp as compared to the on-top site. ${ }^{21}$ When the mixed $p(2 \times 2)_{\mathrm{O}+\mathrm{CO}}$ phase is completed, the STM images basically only show the CO molecules (Fig. 6).

To test this result, STM images were simulated for several mixed structures that appeared reasonable. The models are
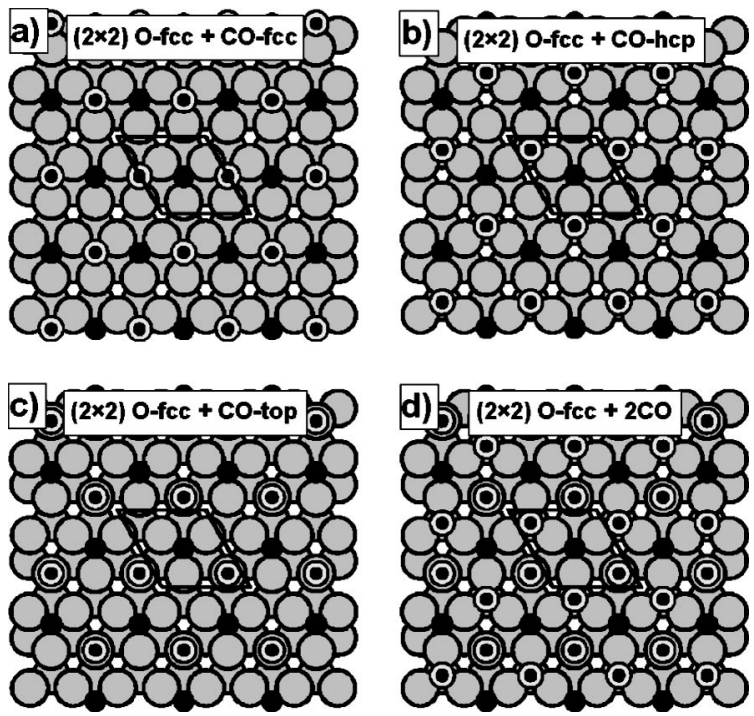

FIG. 12. Models calculated for the $p(2 \times 2)_{\mathrm{CO}+\mathrm{O}}$ phase. (a) Oxygen on fcc and $\mathrm{CO}$ on fcc sites. (b) Oxygen on fcc and CO on hep sites. (c) Oxygen on fcc sites and $\mathrm{CO}$ on top. (d) Oxygen on fcc sites and two CO molecules on hep sites and on top. The simulated STM images are similar for all of these structures. In all of them, only one CO molecule per unit cell is seen [in (d) only the on top].

shown in Fig. 12: (a) O-fcc $+\mathrm{CO}-\mathrm{fcc}$, (b) O-fcc $+\mathrm{CO}-\mathrm{hcp}$, (c) $\mathrm{O}-\mathrm{fcc}+\mathrm{CO}-\mathrm{top}$, (d) O-fcc+CO-hcp+CO-top. The last structure is similar to the $p(2 \times 2)_{3 \mathrm{CO}}$ structure investigated in Ref. 20, but with one oxygen atom and two CO molecules per unit cell. It is found that all of these structures display quite similar contours that are also similar to the experimental STM images. Figure 13 shows a comparison between the experimental image [Fig. 13(a)] and the simulated image for the mixed $p(2 \times 2)_{\mathrm{O}+\mathrm{CO}}$ structure [Fig. 13(b)] with one $\mathrm{CO}$ per unit cell on the hep site [corresponding to the model in Fig. 12(b)]. There is good agreement, so that the proposed model is consistent with the imaging in STM, but the other structures could not have been excluded from the STM contours alone. Differences in the corrugation, $0.25 \AA$ for the experimental STM image and $0.44 \AA$ for the simulated image, can again be attributed to the geometry of the tip apex.
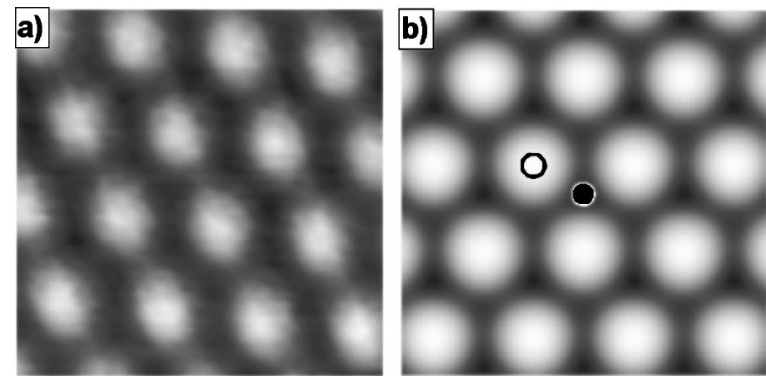

FIG. 13. Comparison between experiment and theory. (a) STM image of the mixed $p(2 \times 2)_{\mathrm{CO}+\mathrm{O}}$ structure $(2 \mathrm{~nm} \times 2 \mathrm{~nm}) ; T$ $=103 \mathrm{~K}, V=-1.7 \mathrm{~V}, I=0.9 \mathrm{nA}$; corrugation is $0.23 \AA$. (b) Simulated image for the CO-hcp model in Fig. 12(b) with the same tunnel conditions. The comparison indicates a good agreement for this model. 


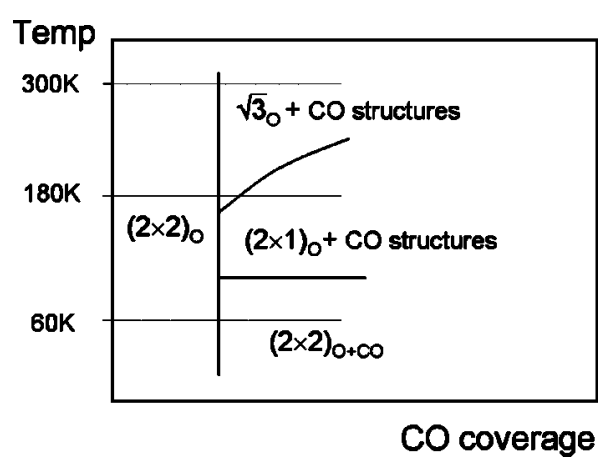

FIG. 14. Tentative temperature vs CO coverage phase diagram with the different structures observed in this study.

\section{DISCUSSION}

The various structures that were resolved by STM during adsorption of $\mathrm{CO}$ on the oxygen covered $\mathrm{Pd}(111)$ surface can be mapped out in a temperature vs $\mathrm{CO}$ coverage phase diagram (Fig. 14). The indicated mixed $\mathrm{O}+\mathrm{CO}$ phases are not thermodynamically stable with respect to formation of $\mathrm{CO}_{2}$, which is, however, sufficiently slowed down below $300 \mathrm{~K}$ to observe the phase transitions. There are also other stabilizing kinetic effects, in particular the surface diffusion of $\mathrm{O}$ atoms.

In the absence of $\mathrm{CO}$ and in the beginning of the $\mathrm{CO}$ adsorption the known $p(2 \times 2)_{\mathrm{O}}$ structure was resolved. By adsorption of oxygen at temperatures below $300 \mathrm{~K}$ the order of the $p(2 \times 2)_{\mathrm{O}}$ structure could be improved. STM images recorded at $176 \mathrm{~K}$ (Fig. 7) show only a few hopping events between consecutive images, so the atoms are not completely immobile, but diffusion processes are slow at this temperature. At still lower temperatures (below $130 \mathrm{~K}$ ) the diffusion of oxygen adatoms is too slow to form an ordered $p(2$ $\times 2)_{\mathrm{O}}$ structure.

$\mathrm{CO}$ adsorption at room temperature causes a phase transition of the $(2 \times 2)_{\mathrm{O}}$ into the $(\sqrt{3} \times \sqrt{3}) R 30_{\mathrm{O}}^{\circ}$ structure. Combined TDS and LEED experiments show that the transition is directly correlated with the reaction. Before completion of the transition the $\mathrm{CO}_{2}$ production stays very low. This effect corresponds to the known induction phase that precedes the onset of the reaction. When the entire oxygen layer has been compressed in the $(\sqrt{3} \times \sqrt{3}) R 30_{\mathrm{O}}^{\circ}$ structure, the $\mathrm{CO}_{2}$ formation rate increases rapidly. The following maximum and decreasing reaction rate is simply resulting from the decreasing coverage of oxygen atoms. The surface finishes covered with $\mathrm{CO}$ as the formed $\mathrm{CO}_{2}$ desorbs immediately.

An induction phase for this reaction has been reported before. ${ }^{11}$ However, this earlier work suggested that a constant $\mathrm{CO}$ exposure is connected with the maximum of the $\mathrm{CO}_{2}$ rate (0.6L in Ref. 11 and $0.56 \mathrm{~L}$ in Ref. 45 ). By contrast, the present investigation shows that a constant exposure is connected with the completion of the induction period. This exposure $(0.55 \mathrm{~L})$ at the same time corresponds to a maximum $(1 / 3,1 / 3)$ LEED spot intensity, which according to Fig. 2(c) reflects a $(\sqrt{3} \times \sqrt{3}) R 30_{\mathrm{O}}^{\circ}$ covered surface. Seitsonen et al. also reported an induction phase at a fixed dose $(0.7 \mathrm{~L}) .^{21}$ It can be concluded that the $p(2 \times 2)_{\mathrm{O}}$ to $(\sqrt{3}$ $\times \sqrt{3}) R 30_{\mathrm{O}}^{\circ}$ transition is indeed a critical step before the re- action at $300 \mathrm{~K}$. The reacting phase is the $(\sqrt{3} \times \sqrt{3}) R 30_{\mathrm{O}}^{\circ}$ rather than the $p(2 \times 2)_{\mathrm{O}}$ structure, which can be explained by the lower stability of this compressed phase and the resulting lower activation energy for the formation to give $\mathrm{CO}_{2}$.

At lower temperatures, at around $170 \mathrm{~K}$, the $(2 \times 2)_{\mathrm{O}}$ to $(\sqrt{3} \times \sqrt{3}) R 30_{\mathrm{O}}^{\circ}$ phase transition is accompanied by bright features appearing first within the $(2 \times 2)_{\mathrm{O}}$ phase [Fig. 4(b)] and then at the borders of still intact $(2 \times 2)_{\mathrm{O}}$ domains [Fig. 4(c)]. An obvious interpretation is that these features are $\mathrm{CO}$ molecules located at adsorption sites directly near $\mathrm{O}$ atoms, most likely in some confined geometry, where they are immobilized and become resolved by STM.

That an oxygen $(\sqrt{3} \times \sqrt{3}) R 30^{\circ}$ phase was observed [Fig. $4(\mathrm{~d})]$ and the reported $(\sqrt{3} \times \sqrt{3}) R 30^{\circ}$ structure of the $\mathrm{CO}$ domains ${ }^{20}$ evidence that both phases form separated domains. Previous experiments ${ }^{11}$ and theoretical calculations ${ }^{21}$ agree that these phases are separated.

At low temperatures $\mathrm{CO}$ adsorption causes a second phase transition, into a $(2 \times 1)$ phase, which was also resolved by STM. As already pointed out, the STM simulations favor a pure oxygen structure, at variance with conclusions from previous experiments, ${ }^{11,45}$ but in agreement with recent DFT calculations. ${ }^{21}$ An interesting aspect of this phase is the shapes of the islands, which are irregular directly after formation [Fig. 4(e)], but then develop a pronounced elongation perpendicular to the rows of the close-packed $\mathrm{O}$ atoms [Fig. 4(f)]. This aspect ratio is exactly opposite to other surface phases involving atomic rows, e.g., many of the reconstructions of the fcc-metal (110) surfaces. ${ }^{1}$ These are mostly characterized by anisotropic islands with the long sides parallel to the atomic rows. An explanation is that there are repulsive interactions between the $\mathrm{O}$ atoms in the $(2 \times 1)_{\mathrm{O}}$ structure on $\operatorname{Pd}(111)$, as follows from the fact that it forms only by compression. Moreover, the $\mathrm{O}$ adsorption energy is by $0.31 \mathrm{eV}$ lower than for the $(2 \times 2)_{\mathrm{O}}$ structure. ${ }^{21}$ The system can relieve some of the strain along the close-packed rows by forming narrow islands with short rows. STM data about the reaction of this phase with $\mathrm{CO}$ will be presented in a separate paper. $^{41}$

At temperatures between 60 and $110 \mathrm{~K}$ a new surface phase was observed: the mixed $p(2 \times 2)_{\mathrm{O}+\mathrm{CO}}$ structure. At these low temperatures the reaction to give $\mathrm{CO}_{2}$ is completely suppressed. In addition the $\mathrm{O}$ atoms are no longer mobile, so that the phase transitions at the higher temperatures are also suppressed. However, the $\mathrm{CO}$ molecules are still mobile, so they can occupy defined adsorption sites between the $\mathrm{O}$ atoms and form a well-ordered overlayer. The hcp site of the $\mathrm{CO}$ molecules agrees with the general trend of CO to occupy hollow sites on the $\operatorname{Pd}(111)$ surface. ${ }^{21}$ The brightness variations of $\mathrm{CO}$ molecules in the immediate neighborhood of point defects (Fig. 6) can be understood as an electronic imaging effect, caused, e.g., by a charge shift between some foreign atom and the surrounding $\mathrm{CO}$ molecules.

\section{CONCLUSIONS}

Adsorption of $\mathrm{CO}$ on a $(2 \times 2)$ oxygen covered $\mathrm{Pd}(111)$ surface was studied by means of STM, LEED, and mass 
spectrometry at temperatures between 60 and $300 \mathrm{~K}$. The investigation aimed at the structures of the different coadsorbed phases and the transitions between the (metastable) phases. Our main conclusion is that the structures play an important role in the reaction to give $\mathrm{CO}_{2}$. We have observed an induction phase at room temperature where the structures of the adsorbates become compressed before the reaction starts. Therefore, the classical Langmuir-Hinshelwood mechanism is insufficient for describing the catalytic reaction of these complex systems. Resolved adsorbate structures were the $(2 \times 2)_{\mathrm{O}}$, the $(\sqrt{3} \times \sqrt{3}) R 30_{\mathrm{O}}^{\circ}$, the $c(4 \times 2)_{\mathrm{CO}}$, the $p(2 \times 1)_{\mathrm{O}}$ and a so far unknown mixed $p(2 \times 2)_{\mathrm{O}+\mathrm{CO}}$ structure. The reactivity of these structures is discussed in another paper. $^{41}$

The $(2 \times 2)_{\mathrm{O}}$ phase is formed by relatively mobile $\mathrm{O}$ atoms. At $300 \mathrm{~K}$ the mobility prevents resolution of hopping events of individual atoms even at imaging rates of 20 frames per sec, but the ordered $(2 \times 2)_{\mathrm{O}}$ structure could be resolved. At $170 \mathrm{~K}$ the mobility of this phase is almost frozen in. The $\mathrm{O}$ atoms are imaged dark with a normal-contrast metal tip and bright with a reverse-contrast $\mathrm{O}$ atom tip.

The $(\sqrt{3} \times \sqrt{3}) R 30_{\mathrm{O}}^{\circ}$ phase is formed by adsorption of $\mathrm{CO}$ on the $(2 \times 2)_{\mathrm{O}}$ phase between $300 \mathrm{~K}$ and approximately 170 $\mathrm{K}$. The data indicate that this compressed phase consists only of $\mathrm{O}$ atoms, the $\mathrm{CO}$ molecules form separate domains between the $\mathrm{O}$ islands. In the STM images this structure displays the same contrast inversion as the $(2 \times 2)_{\mathrm{O}}$ structure when the tip changes between metallic and $\mathrm{O}$ terminated. At room temperature the formation of this phase and the onset of the reaction to give $\mathrm{CO}_{2}$ occur simultaneously.

The $(2 \times 1)_{\mathrm{O}}$ phase is formed by adsorption of $\mathrm{CO}$ at around $170 \mathrm{~K}$. Simulations of the STM images indicate that this more strongly compressed structure is formed only by $\mathrm{O}$ atoms. Mixed $\mathrm{O}+\mathrm{CO}$ structures that were suggested by previous investigations are not confirmed. The $(2 \times 1)_{\mathrm{O}}$ domains display characteristic elongated shapes perpendicular to the onefold period, which can be understood from the repulsion between the $\mathrm{O}$ atoms.

The only mixed $\mathrm{O}+\mathrm{CO}$ phase is the $p(2 \times 2)_{\mathrm{O}+\mathrm{CO}}$ structure that occurs by adsorption of $\mathrm{CO}$ at temperatures between 60 and $110 \mathrm{~K}$. The STM data show that the CO molecules occupy the hcp sites between the $\mathrm{O}$ atoms, consistent with the simulated STM images. The stability of this phase is explained by the suppressed reaction and by the immobility of the $\mathrm{O}$ atoms, preventing the phase separations observed at higher temperatures.

\section{ACKNOWLEDGMENTS}

We acknowledge J.A. Martín-Gago, R. Pérez, and E. Valbuena for their critical reading and comments. J.M. acknowledges the Spanish Ministerio de Ciencia, Project No. MAT2002-00395. S.H.K. thanks the German Academic Exchange Service (DAAD) for financial support. The work of J.C. was supported by the Spanish DGICyT under Contract No. MAT2001-1956.
*Electronic address: jmendez@icmm.csic.es

†Present address: Lawrence Berkeley National Laboratory, Materials Sciences Division, Berkeley, CA.

Fresent address: Universität München, Department Chemie, Germany.

${ }^{1}$ J. Wintterlin, Adv. Catal. 45, 131 (2000).

${ }^{2}$ J. Wintterlin, S. Völkening, T. Janssens, T. Zambelli, and G. Ertl, Science 278, 1931 (1997).

${ }^{3}$ S. Völkening and J. Wintterlin, J. Chem. Phys. 114, 6382 (2001).

${ }^{4}$ X. Guo and J. T. Yates Jr., J. Chem. Phys. 90, 6761 (1989).

${ }^{5}$ T. Gießel, O. Schaff, C. J. Hirschmugl, V. Fernandez, K. M. Schindler, A. Theobald, S. Bao, R. Lindsay, W. Berndt, A. M. Bradshaw, C. Baddeley, A. F. Lee, R. M. Lambert, and D. P. Woodruff, Surf. Sci. 406, 90 (1998).

${ }^{6}$ T. Engel, J. Chem. Phys. 69, 373 (1978).

${ }^{7}$ H. Conrad, G. Ertl, J. Küppers, and E. E. Latta, Surf. Sci. 65, 245 (1977).

${ }^{8}$ A. Steltenpohl and N. Memmel, Surf. Sci. 443, 13 (1999).

${ }^{9}$ F. P. Leisenberger, G. Koller, M. Sock, S. Surnev, M. G. Ramsey, F. P. Netzer, B. Klötzer, and K. Hayek, Surf. Sci. 445, 380 (2000).

${ }^{10}$ G. Zheng and E. I. Altman, Surf. Sci. 462, 151 (2000).

${ }^{11}$ H. Conrad, G. Ertl, and J. Küppers, Surf. Sci. 76, 323 (1978).

${ }^{12}$ G. Ertl and J. Koch, Z. Naturforsch. A 25A, 1906 (1970).

${ }^{13}$ A. M. Bradshaw and F. M. Hoffmann, Surf. Sci. 78, 513 (1978).

${ }^{14}$ M. Tüshaus, W. Berndt, H. Conrad, A. M. Bradshaw, and B. Persson, Appl. Phys. A: Solids Surf. A51, 91 (1990).
${ }^{15}$ W. K. Kuhn, J. Szanyi, and D. W. Goodman, Surf. Sci. Lett. 274, L611 (1992).

${ }^{16}$ J. Szanyi, W. K. Kuhn, and D. W. Goodman, J. Vac. Sci. Technol. A 11, 1969 (1993).

${ }^{17}$ D. Loffreda, D. Simon, and P. Sautet, Surf. Sci. 425, 68 (1999).

${ }^{18}$ I. Zasada and M. A. Van Hove, Surf. Sci. Lett. 457, L421 (2000).

${ }^{19}$ P. Sautet, M. K. Rose, J. C. Dunphy, S. Behler, and M. Salmeron, Surf. Sci. 453, 25 (2000).

${ }^{20}$ M. K. Rose, T. Mitsui, J. C. Dunphy, A. Borg, D. F. Ogletree, M. Salmeron, and P. Sautet, Surf. Sci. 512, 48 (2002).

${ }^{21}$ A. P. Seitsonen, Y. D. Kim, S. Schwegmann, and H. Over, Surf. Sci. 468, 176 (2000).

${ }^{22}$ G. Odörfer, E. W. Plummer, H. J. Freund, H. Kuhlenbeck, and M. Neumann, Surf. Sci. 198, 331 (1988).

${ }^{23}$ H. Brune, J. Wintterlin, J. Trost, and G. Ertl, J. Chem. Phys. 99, 2128 (1993).

${ }^{24}$ J. Wintterlin, J. Trost, S. Renisch, R. Schuster, T. Zambelli, and G. Ertl, Surf. Sci. 394, 159 (1997).

${ }^{25}$ J. Méndez and H. Niehus, Appl. Phys. Lett. 142, 152 (1999).

${ }^{26}$ A. Böttcher, H. Niehus, S. Schwegmann, H. Over, and G. Ertl, J. Phys. Chem. B 101, 11185 (1997).

${ }^{27}$ J. Cerdá and F. Soria, Phys. Rev. B 61, 7965 (2000).

${ }^{28}$ J. M. Soler, E. Artacho, J. Gale, A. García, J. Junquera, P. Ordejón, and D. Sánchez-Portal, J. Phys.: Condens. Matter 14, 2745 (2002).

${ }^{29}$ N. Troullier and J. L. Martins, Phys. Rev. B 43, 1993 (1991).

${ }^{30}$ J. Cerdá, M. A. Van Hove, P. Sautet, and M. Salmeron, Phys. Rev. 
B 56, 15885 (1997).

${ }^{31}$ See also www.icmm.csic.es/jcerda for additional information about the GREEN code.

${ }^{32}$ C. I. Carlisle, D. A. King, M.-L. Bocquet, J. Cerdá, and P. Sautet, Phys. Rev. Lett. 84, 3899 (2000).

${ }^{33}$ J. Cerdá, A. Michaelides, M.-L. Bocquet, P. J. Feibelman, T. Mitsui, M. Rose, E. Fomine, and M. Salmeron, Phys. Rev. Lett. 93, 116101 (2004).

${ }^{34}$ J. P. Perdew, J. A. Chevary, S. H. Vosko, K. A. Jackson, M. R. Pederson, D. J. Singh, and C. Fiolhais, Phys. Rev. B 46, 6671 (1992).

${ }^{35}$ D. M. Ceperley and B. J. Alder, Phys. Rev. Lett. 45, 566 (1980).

${ }^{36}$ S. Datta, Transport in Mesoscopic Systems (Cambridge University Press, Cambridge, UK, 1995).

${ }^{37}$ J. Wintterlin, R. Schuster, and G. Ertl, Phys. Rev. Lett. 77, 123 (1996)
${ }^{38}$ P. Sautet, Surf. Sci. 374, 406 (1997).

${ }^{39}$ J. M. Blanco, C. González, P. Jelínek, J. Ortega, F. Flores, R. Pérez, M. Rose, M. Salmerón, J. Méndez, J. Wintterlin, and G. Ertl, Phys. Rev. B (to be published).

${ }^{40}$ F. Calleja, A. Arnau, J. J. Hinarejos, A. L. Vázquez, W. A. Hofer, P. M. Echenique, and R. Miranda, Phys. Rev. Lett. 92, 206101 (2004).

${ }^{41}$ S. H. Kim, J. Méndez, J. Wintterlin, and G. Ertl (unpublished).

${ }^{42}$ R. Imbihl and J. E. Demuth, Surf. Sci. 173, 395 (1986).

${ }^{43}$ M.-L. Bocquet, J. Cerdá, and P. Sautet, Phys. Rev. B 59, 15437 (1999).

${ }^{44}$ D. Loffreda, D. Simon, and P. Sautet, J. Chem. Phys. 108, 6447 (1998).

${ }^{45}$ T. Matsushima and H. Asada, J. Chem. Phys. 85, 1658 (1986).

${ }^{46}$ J. M. Blanco, C. González, P. Jelínek, J. Ortega, F. Flores, and R. Pérez, Phys. Rev. B 70, 085405 (2004). 